\title{
Wetting and dewetting of extracellular matrix and glycocalix models
}

\author{
Motomu Tanaka ${ }^{1}$, Florian Rehfeldt, Matthias F Schneider, \\ Gerald Mathe, Antero Albersdörfer, Klaus R Neumaier, \\ Oliver Purrucker and Erich Sackmann \\ Biophysics Laboratory, Department of Physics, Technical University Munich, D-85748 Garching, \\ Germany \\ E-mail: mtanaka@ph.tum.de
}

Received 24 March 2004

Published 18 February 2005

Online at stacks.iop.org/JPhysCM/17/S649

\begin{abstract}
In this paper, we study wetting and dewetting of hydrated biopolymer layers mediating cell-cell and cell-tissue contacts, called the extracellular matrix and cell surface glycocalix, by the combination of various physical techniques. Here, the sum of the net effects of the various interfacial forces, which is referred to as the disjoining pressure, is used as a semi-quantitative measure to describe the thermodynamics of hydrated interlayers. The disjoining pressure can be measured by applying external forces to maintain the equilibrium distance between two parallel surfaces (in biology, two neighbouring plasma membranes). Using artificial models of the extracellular matrix and glycocalix, we describe stable cell-cell contacts in terms of the wetting (or spreading) of complex fluids on polymer surfaces. In fact, the adjustment of the wetting interaction via thin hydrating layers enables us to transform three-dimensional cell membranes into quasi-two-dimensional films on macroscopically large surfaces. Fine-tuning of local wetting conditions at the interface further allows for the selective wetting of native cell membranes on microstructured polysaccharide films, which has a large potential for individual detection of biological functions in confined geometries.
\end{abstract}

(Some figures in this article are in colour only in the electronic version)

\section{Introduction and theoretical background}

Interactions between cells and tissues in nature are mediated by a complex interplay of shortrange and long-range forces across hydrated layers of biopolymers, such as the extracellular

1 Author to whom any correspondence should be addressed. On leave at: Department of Physics and Centre of Excellence, Kyoto University, Japan. 
(a)

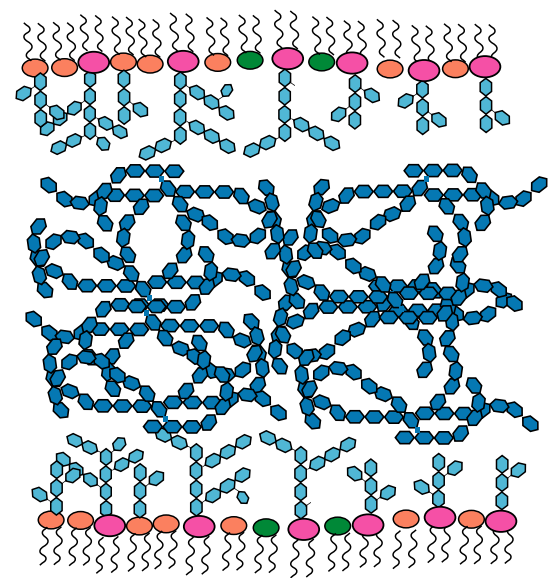

(b)

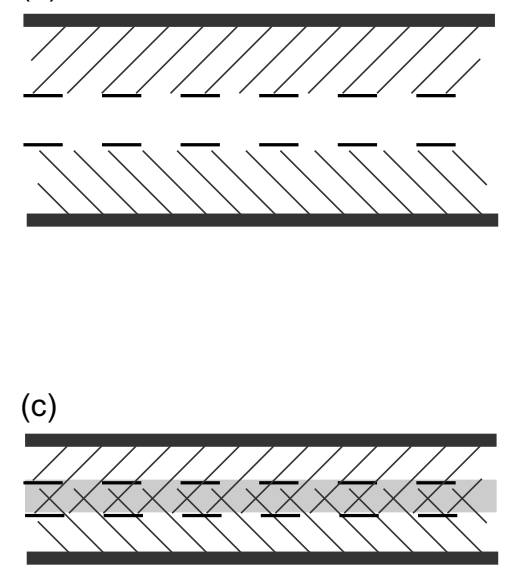

Figure 1. (a) A schematic illustration of a cell-cell contact mediated by a hydrated biopolymer layer (glycocalix and extracellular matrix). (b) Two parallel Gibbs layers, where the intrinsic properties of the bulk phase are retained. At equilibrium, there is no work required to change the interlayer distance $d$. (c) Two parallel planes, where the long-range force fields overlap within the interlayer. Here, either positive or negative work is required to change the interlayer distance; i.e. the pressure within the interlayer differs from that in the bulk phase (disjoining pressure).

matrix (ECM) [1] and cell surface glycocalix [2]. They maintain a certain distance (typically in the range of $10-100 \mathrm{~nm}$ ) between neighbouring cells to avoid direct, non-specific cell-cell contacts as well as to create hydrodynamic pathways for solute transport (figure 1(a)).

If one considers two planes (in this case, two plasma membranes) maintaining a stable, distinct separation distance via a thin interlayer, there are two possible scenarios. If the middle of a thin interlayer in equilibrium retains its intrinsic bulk properties, the individual interfaces can be explained within the framework of the classical Gibbs capillary theory (figure 1(b)). Here, a decrease in the interlayer thickness at constant volume and constant interface areas does not cost any change in the free energy of the system (i.e. the pressure in the interlayer is equal to zero) except for the energy dissipation to overcome passive resistance forces such as a viscous force. This ceases to hold as soon as the fields of the long-range forces in thin interlayers overlap (figure 1(c)) [3]. Under these conditions, any change in the interlayer thickness will cost positive or negative work. This work originates from the net sum of attractive or repulsive forces in the overlapped interfacial region, which can range over even more than several tens of nanometres. The long-range forces operating in thin interlayers can be specified as van der Waals (dispersion) forces, electrostatic forces, steric (entropic) forces, and hydration forces. The last of these, the hydration force, was found to stabilize colloidal dispersions [4, 5], polar surfaces [6], and soap films [7]. This force is a consequence of the work necessary for removing water from a hydrated layer to the bulk liquid phase.

To describe the thermodynamics of thin liquid films analytically, Derjaguin introduced the concept of a disjoining pressure, which is the sum of the net effects of the various interfacial forces $[3,4]$. This approach is very helpful for simplifying the extremely complex calculation of each force contribution. The disjoining pressure $\Pi$ can be measured by applying external forces to keep the interlayer at mechanical equilibrium. That is, it can be given as the difference between the pressure in the interlayer $P_{1}$ and the pressure in the bulk layer $P_{0}$ :

$$
\Pi=P_{1}-P_{0} .
$$


The disjoining pressure can also be related to other thermodynamic parameters. For example, for a change in the interlayer thickness $d$ by $\delta d$ at constant temperature $T$ and chemical potential $\mu$, the work done by the external force $-\Pi(d) \partial d$ is equal to the increment of the Gibbs free energy $\delta G$ :

$$
\Pi(d)=-(\partial G / \partial d)_{T, \mu} .
$$

The minimum of the free energy that determines the equilibrium state can be found at $\partial^{2} G / \partial d^{2}>0$. Thus, to keep a specific equilibrium distance $d$ between two planes (in biology, a plasma membrane contact mediated by a biopolymer interlayer), the disjoining pressure should fulfil the condition

$$
\partial \Pi(d) / \partial d<0 .
$$

In the case of cell adhesion, the trapping of membranes at this local minimum can be referred to as 'weak adhesion', where the interaction potential may be approximated by a harmonic potential $[8,9]$.

On the other hand, the interlayer becomes unstable when

$$
\partial \Pi(d) / \partial d>0 \text {. }
$$

Continuous thinning of the wetting layer below the free energy minimum leads to a negative disjoining pressure, resulting in dewetting of the film. Typical examples are the rupturing of polymer films and that of surfactant films [10-12]. In biological systems, this can be found as the formation of tight membrane contacts (adhesion plaques) [13, 14].

In fact, Bell, Dembo, and Bongrad developed a theoretical model of cell adhesion that describes the thermodynamics of adhesion in terms of the lateral osmotic pressure from ligand/receptor pairs $[15,16]$. If one implements the influence of 'mobile' repellers (using lipids with poly(ethylene glycol) (PEG) brushes as a simplified glycocalix model) in such a model, the chemical equilibrium between the repellers in the tight membrane contact zone (called the adhesion plaque) and those in the bulk reservoir must be taken into account [14]. This revised model predicts a double-well potential with a shallow minimum from 'weak adhesion' and a sharp minimum inside the repeller, which was confirmed by recent experimental work [17]. This minimum at a short distance (dominated by van der Waals attraction) becomes dominant when the van der Waals attraction exceeds this repeller pressure. If one generalizes a cell membrane as a complex fluid film, the discontinuous transition between 'weak adhesion' and 'strong adhesion' can be interpreted as a first-order wetting/dewetting transition [13].

To further apply such a 'wetting principle' to describe cell-cell and cell-tissue interactions, well defined, realistic models of the ECM and glycocalix are required. In addition to the scientific impact in studying the influence of the chemical nature and morphology of polymers on cell-cell and cell-tissue interactions, such biocompatible polymer films can also be used practically for proliferation and stress-free immobilization of cells on solid-based devices. One of the promising strategies for quantitative characterization of ECM and glycocalix models is the confinement of hygroscopic polymer films or monolayers of lipopolymers (or glycolipids) at interfaces. To date, grafted films of dextran [18] and poly(ethylene glycol) (PEG) brushes [19] have been used to achieve a sufficient resistance against non-specific adsorption of proteins, which has been used in numerous fields.

In this paper, we introduce several interdisciplinary approaches in order to study wetting and dewetting of ECM and glycocalix models by the combination of experiments in equilibrium and dynamic swelling experiments. As synthetic ECM models, we prepare several kinds of ultrathin films (dry film thickness of 2-200 nm) of polysaccharides (dextran, hyaluronic acid, and cellulose) and end-grafted polymer brushes. Here, the disjoining pressure, i.e., the first derivative of the interaction potential with respect to the thickness of the wetting layer, can 
be used as a quantitative measure for the force operating within such hydrated biopolymer films [20]. To physically model the cell surface glycocalix, monolayers of synthetic glycolipids and lipopolymers confined at the air/water or solid/air interface are chosen as the model systems.

As a non-invasive technique with a high thickness resolution $( \pm 0.1 \AA)$ for studying relationships between thickness and osmotic pressure within the hydrated films, we use an ellipsometer coupled to a climate chamber [21-23]. Here, the model ECM layers are deposited onto solid substrates and are exposed to humid atmosphere. This technique allows the measurement of equilibrium film thickness as a function of relative atmospheric humidity and, therefore, one can gain quantitative force-distance relationships. The fast kinetics of the water uptake can be analysed by plotting film thickness as a function of time across an abrupt change of the relative humidity (osmotic shock). Furthermore, as a preliminary study on the viscoelastic properties of hydrated polymer films, a colloidal probe technique coupled to a microinterferometer is applied to determine the equilibrium layer thickness, the interfacial interaction potential, and the characteristic mesh size of the charged polysaccharide (hyaluronic acid) film. In the second part, we highlight some of our recent studies on wetting of biological membranes on homogeneous and microstructured polymer films. In spite of experimental difficulties, the results obtained qualitatively support our 'wetting hypothesis', namely, that a stable membrane-surface contact should fulfil the complete wetting conditions in slightly repulsive interfacial potentials. Details of the results will be discussed in the following sections.

\section{Static and dynamic hydration of ECM models}

\subsection{Equilibrium hydration of ultrathin polymer films}

Natural polysaccharides, dextran from Leuconostoc mesenteroides (molar mass $\sim 0.5 \mathrm{MDa}$ ) and bacterial hyaluronic acid from Streptococcus zooepidemicus (molar mass $\sim 1.0 \mathrm{MDa}$ ), are deposited by spin coating of aqueous solutions of polysaccharides [21]. The thickness of the films can be controlled between 15 and $200 \mathrm{~nm}$ by adjusting the concentration of polysaccharides. Although these physisorbed films are not stable in the presence of bulk water, they show sufficient stability for the experiments in humid atmosphere. Ultrathin films of trimethylsilylcellulose are deposited by the Langmuir-Blodgett (LB) method as well as by spin coating [24, 25]. After the deposition, trimethylsilyl side chains are regenerated to hydroxyl groups by treatment with acid vapour. It should be noted that the regenerated cellulose films are stable in the presence of bulk water due to the tight hydrogen bonding network between rod-like cellulose backbones, which is in contrast to the case for physisorbed films of other polysaccharides (dextran and hyaluronic acid). Films of poly(2-oxazoline) derivatives with trimethoxysilane coupling groups are prepared by covalent grafting onto $\mathrm{SiO}_{2}$ surfaces, which enables the film to be stable in bulk water [22]. Poly(2-oxazoline) derivatives synthesized by living cationic polymerization possess well defined degrees of polymerization and polydispersity indices and, furthermore, different side substitutions enable us to tailor the hydrophilicity of the chains. Prior to the swelling experiments, we confirm the smooth topography of the polymer films, exhibiting root mean square roughness values of $4-6 \AA$ within a $1 \times 1 \mu \mathrm{m}^{2}$ AFM scan (tapping mode) [22, 25].

The sample environment for the static hydration experiments is presented schematically in figure 2(a). The sample deposited on a silicon wafer (covered with a thermal oxide layer with thickness of about $150 \mathrm{~nm}$ ) reaches an equilibrium in a self-built climate chamber, at which the temperature $(293 \mathrm{~K})$ and the relative humidity (4-98\%) are precisely controlled. It should be noted that our experimental system does not possess mirror symmetry, in contrast to the symmetric parallel geometry in figure 1 . Therefore, the theoretical treatments need to be 

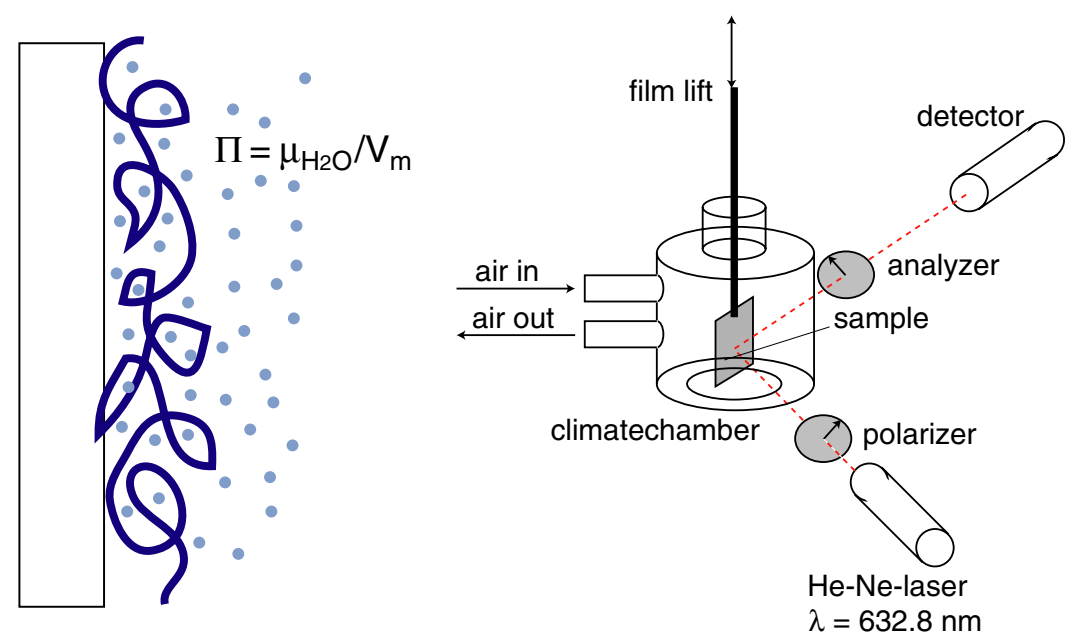

Figure 2. (a) A schematic view of the sample environment. Here the disjoining pressure can be related to the chemical potential of water in the film with respect to that in the bulk, $\Pi(d)=-\mu_{\mathrm{H}_{2} \mathrm{O}} / V_{\mathrm{m}}$. (b) An ellipsometer coupled with a climate chamber. Atmospheric relative humidity can be controlled between 4 and $98 \%$.

multiplied by a factor of the order of unity. The coupling to an ellipsometer (figure 2(b)) [2123] allows the static hydration of the film to be studied by measuring the equilibrium film thickness. As the atmospheric humidity is carefully maintained below the condensation limit (i.e. the sample contains no bulk water), the chemical potential of water within the polymer film with respect to that of bulk water can be represented as

$$
-\mu_{\mathrm{H}_{2} \mathrm{O}}=-R T \ln \left(\frac{p_{\mathrm{w}}}{p_{0}}\right)
$$

where $p_{\mathrm{w}} / p_{0}$ is the ratio between the actual vapour pressure and the saturation pressure. Thus, the disjoining pressure that counterbalances the osmotic pressure can be given as

$$
\Pi(d)=-\frac{\mu_{\mathrm{H}_{2} \mathrm{O}}}{V_{\mathrm{m}}}=-\left(\frac{R T}{V_{\mathrm{m}}}\right) \ln \left(\frac{p_{\mathrm{w}}}{p_{0}}\right)=-\left(\frac{R T}{V_{\mathrm{m}}}\right) \ln X
$$

where $V_{\mathrm{m}}$ is the molar volume of water and $X$ is the relative humidity [26]. Thus, we introduce an empirical description of the relationship between the net sum force (disjoining pressure) and the film thickness.

The force-distance relationship at high pressure conditions (typically, $\Pi>5 \times 10^{7} \mathrm{~Pa}$ ), i.e., low humidity conditions, exhibited a power-law potential [20]

$$
\Pi(d) \propto d^{-n} .
$$

Under these conditions, the film is strongly compressed, so a very large exponent $(n>15)$ can be obtained. Such a strong repulsion can be approximated with a theta function between hard core particles, reflecting the infinite jump of the energy for a hard core potential that originates from the incompressibility of the polymer chains. In contrast, the force-distance relations in low pressure regimes show an exponential decay, except for the points in the vicinity of the swelling limit. This enables us to compare the hydration of different polymer films in terms of the monomer distance $L$ and the calculated decay length $\lambda_{0}$. In a case where the monomer size is not given, one can use the normalized swelling ratio $\rho$ and the dimensionless decay constant $\lambda^{*}[27,28]$ :

$$
P=P_{0} \exp \left(-\frac{L}{\lambda_{0}}\right)
$$




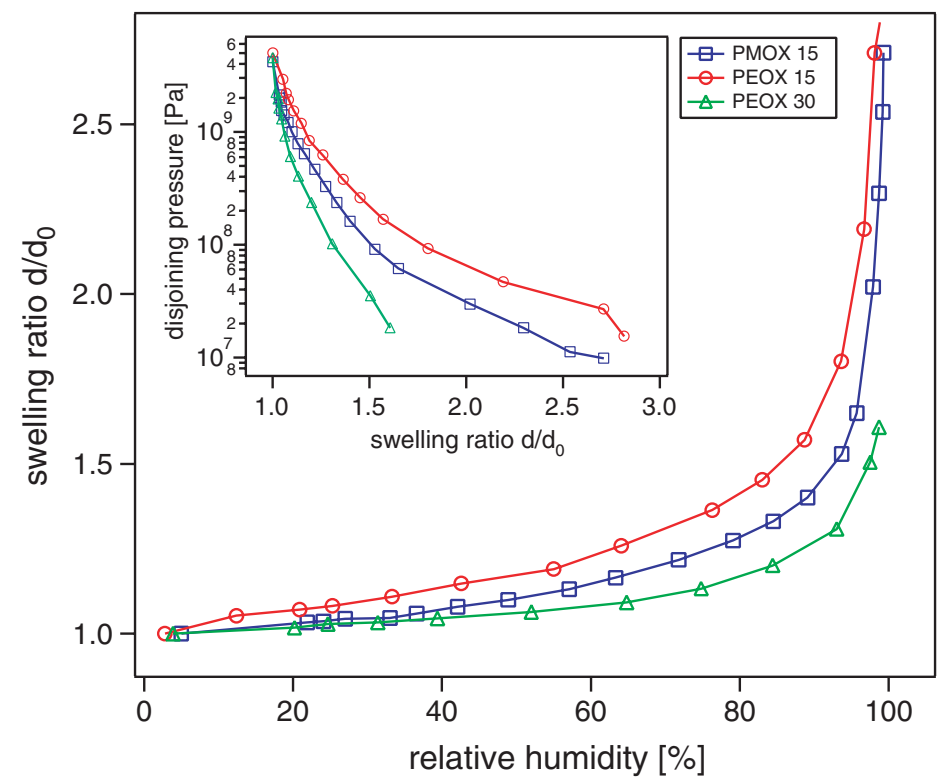

Figure 3. Equilibrium hydration curves for the grafted poly(2-oxazoline) films. The swelling ratio $\rho=d / d_{0}$ is plotted as a function of relative humidity. The force-distance relationship (disjoining pressure versus swelling ratio) is given in the inset.

Table 1. The dry film thickness $d_{0}$ and decay constant $\lambda^{*}$ of the grafted poly(2-oxazoline) films calculated in the disjoining pressure regime, $P<1 \times 10^{7} \mathrm{~Pa}$.

\begin{tabular}{lll}
\hline Polymer & $d_{0}(\AA)$ & $\lambda^{*}$ \\
\hline PMOX15 & $18.2-21.7$ & $0.49-0.56$ \\
PEOX15 & $12.4-20.8$ & $0.48-0.63$ \\
PEOX30 & $24.4-39.7$ & $0.16-0.18$ \\
\hline
\end{tabular}

or

$$
P=P_{0} \exp \left(-\frac{\rho}{\lambda^{*}}\right)
$$

The distance between two neighbouring monomers $L$ can be represented as $L(\rho)=a_{0}\left(\rho^{\frac{1}{3}}-1\right)$ for a cubic lattice model. Here, $a_{0}$ is the size of a monomer and $\rho$ is the swelling ratio.

The swelling curves (i.e., the swelling ratio $\rho$ plotted as a function of the relative humidity $X$ ) of poly(2-methyl-2-oxazoline) (PMOX) and poly(2-ethyl-2-oxazoline) (PEOX) films with different initial thicknesses (figure 3) exhibit a clear dependence upon the chain length [22]. On the other hand, as summarized in table 1, the swelling behaviours of 2-methyl and 2-ethyl oxazolines with the same polymerization degrees show almost identical maximum swelling ratios $\left(\rho_{\max } \sim 3\right)$ and decay constants $\left(\lambda^{*} \sim 0.5\right)$, suggesting that the water uptake ability of the film is independent of the side chains.

In contrast to the case for the end-grafted polymer brushes, the hydration of cellulose LB films and that of spin-coated films at equilibrium are found to be independent of the initial dry film thickness $\left(d_{0}=2.5-18 \mathrm{~nm}\right)$, and surprisingly similar as regards the maximum swelling ratio $\left(\rho_{\max } \sim 1.6\right)$ and the exponential decay length $\left(\lambda_{0}=0.32 \AA\right)$ under low pressure conditions (table 2) [25]. These are 3-6 times smaller than those found for more molten films 


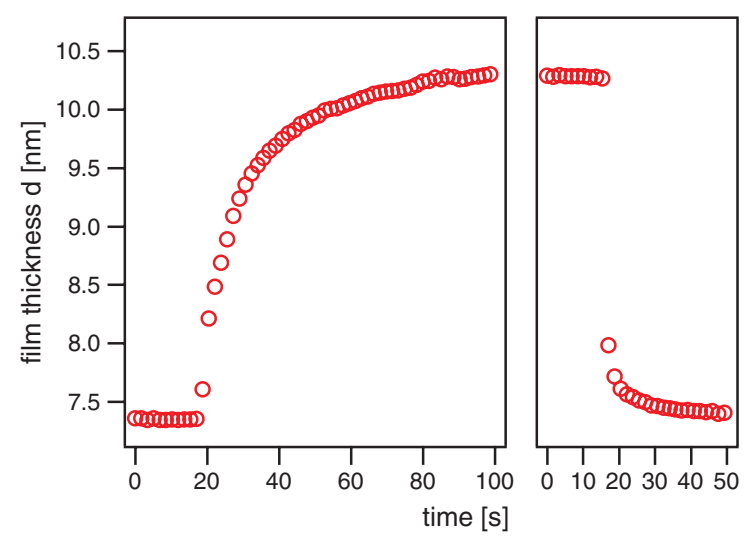

Figure 4. Dynamic hydration/dehydration of a spin-coated cellulose film $\left(d_{0}=73 \AA\right)$, yielding the characteristic time constants $\tau=14 \mathrm{~s}$ (swelling) and $\tau=4 \mathrm{~s}$ (draining), respectively.

Table 2. The dry film thickness $d_{0}$, maximum swelling ratio $\rho_{\max }$, and decay length $\lambda_{0}$ of the regenerated cellulose films, prepared by the Langmuir-Blodgett (LB) method or spin coating (SP), calculated in the disjoining pressure regime, $P<5 \times 10^{7} \mathrm{~Pa}$.

\begin{tabular}{lrll}
\hline Sample & $d_{0}(\AA)$ & $\rho_{\max }$ & $\lambda_{0}(\AA)$ \\
\hline LB-1 & 24 & 1.6 & 0.30 \\
LB-2 & 56 & 1.4 & 0.32 \\
LB-3 & 104 & 1.7 & 0.32 \\
SP-1 & 73 & 1.6 & 0.32 \\
SP-2 & 181 & 1.5 & 0.32 \\
\hline
\end{tabular}

of dextran and hyaluronic acid [21], corresponding to the tighter hydrogen bonding between linear and cylindrical saccharide backbones.

\subsection{Dynamic hydration/dehydration triggered by osmotic pulses}

To study dynamic (i.e., non-equilibrium) hydration/dehydration of polymer thin films, an abrupt change in atmospheric humidity is applied. Monitoring of fast water uptake of hygroscopic polymers is difficult when the finite time required to exchange the atmosphere becomes competitive with (or even longer than) that of the characteristic time constant of hydration. Using the self-made set-up reported elsewhere [25], we can switch the local humidity conditions in the vicinity of the measurement point with a characteristic time of $1 \mathrm{~s}$. Here, we define the characteristic time constant $\tau$ as the time that is required for the film to reach the thickness $d=\left(1-\mathrm{e}^{-1}\right) d_{\max }$ [22].

The time constant calculated from the dynamic hydration of a spin-coated cellulose film (figure 4 left) was $\tau=14 \mathrm{~s} \mathrm{[25].} \mathrm{On} \mathrm{the} \mathrm{other} \mathrm{hand,} \mathrm{the} \mathrm{draining} \mathrm{of} \mathrm{the} \mathrm{film} \mathrm{(figure} 4$ right) seems to be faster than the water uptake, yielding a smaller time constant of $\tau=4 \mathrm{~s}$. It is well worth noting that the kinetic time constants measured for LB films were identical to those for spin-coated films. Interestingly, the characteristic time constant for the hydration of cellulose $(\tau=14 \mathrm{~s})$ is clearly larger than those reported for other polysaccharides with less ordered, more flexible backbones, e.g., $\tau=3 \mathrm{~s}$ for dextran and $\tau=1 \mathrm{~s}$ for hyaluronic acid [21]. Thus, the 'stretched' hydration kinetics of cellulose can be attributed to the less conformational degrees of freedom of 'rigid' cellulose backbones as well as to the strong intermolecular hydrogen bridges for stabilizing their supramolecular architectures under various conditions. 

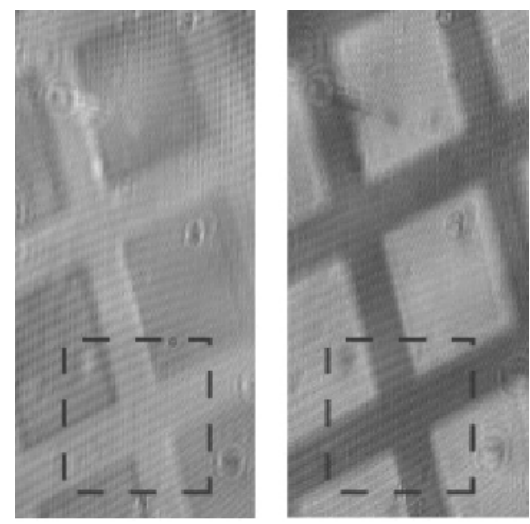

$\overline{40 \mu \mathrm{m}}$

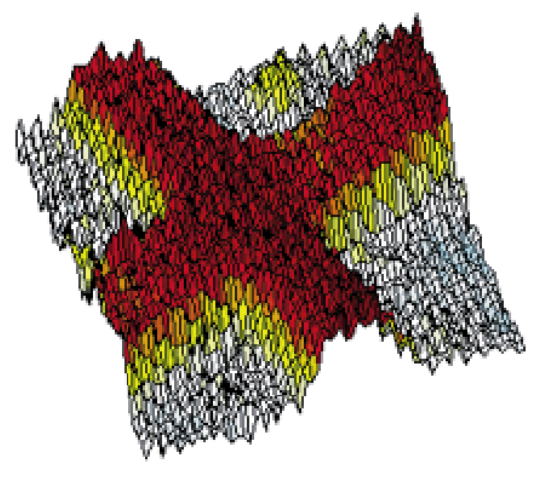

Figure 5. $\Delta$ (left) and $\Psi$ (middle) images of the spin-coated cellulose film ( $d_{0}=183 \AA$ ) taken by the imaging ellipsometer, and the three-dimensional (3D) profiles of the highlighted region (right).

\subsection{Local wetting of structured polymer films}

Wetting of lipid membranes on structured or rough surfaces [29-31] has been described in terms of a general form of the Young equation [32, 33]. Swain and Andelman [9] used the Derjaguin approximation as an analytical tool to theoretically model membrane adhesion on geometrically structured surfaces, such as an isolated cylindrical pit, a single elongated trench, and a periodic array of trenches. Local wetting of heterogeneous substrates with lipid membranes has been studied by deposition of obstacles [34, 35] or fabrication of surfaces with hydrophobic/hydrophilic mismatches [36, 37]. As an alternative approach to fabricating a heterogeneous surface that locally expresses hydrophilic solids and hydrophilic biopolymers, we process the micropatterns of polysaccharide thin films by means of deep UV lithography $[21,25]$. Illumination with deep UV light $(\lambda<220 \mathrm{~nm})$ leads to an ablation of organic molecules, leading to recovery of the original silica surface $[25,38]$. As the first step in monitoring the local water uptake into such patterned polymer films, we study the local wetting (hydration) of the microstructured polysaccharide films. The three-dimensional profiles of the swollen polymer films can be gained in a non-invasive manner using a self-built imaging ellipsometer [39-41]. Light from a He-Ne laser $(\lambda=632.8 \mathrm{~nm})$ is reflected by the sample under an incident angle of $45^{\circ}$ and collected with a microscope objective (numerical aperture NA $=0.4$; magnification: $20 \times$ ). To determine the degree of polarization, the reflected beam passes through a rotatable polarizer, and a tube lens projects the image onto a cooled CCD camera (C4880, Hamamatsu, Germany). Images taken at different angles of polarization enable one to calculate the ellipsometric parameters, $\Psi$ and $\Delta$, for each pixel, leading to a 3D reconstruction (local thickness profile) of the microstructures (figure 5). The local hydration properties of the structured polymer films can be obtained by measurements of ellipsometric images at low and high humidity conditions. The surface heterogeneity can be considered as the columnar heterogeneity, because the height deviation $(5-20 \mathrm{~nm})$ is much smaller than the width of each pattern $(\geqslant 5 \mu \mathrm{m})$. Interestingly, the ultrathin cellulose film shows a strong wetting affinity to the cell membranes, which is completely different from what we found for the bare silica surfaces (see section 3.2). Thus, the control of local wetting conditions is a promising strategy for designing microarrays of native cell membranes in confined geometry. 


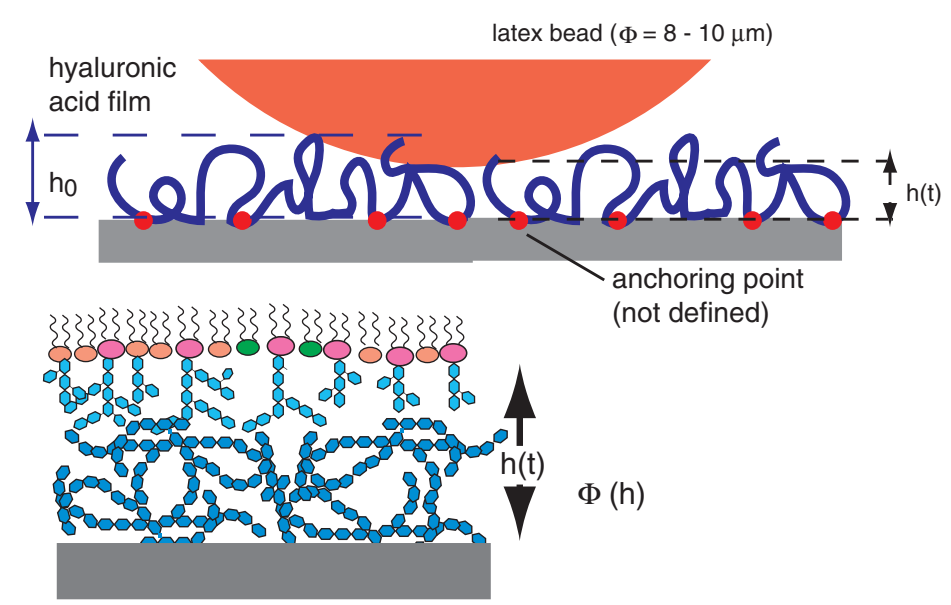

Figure 6. A schematic view of the colloidal probe technique that models the contact between cell membranes and biopolymer films in bulk water. To calculate the interfacial potential as a function of the film thickness $\Phi(h)$ quantitatively, a colloidal probe (with 16-20 $\mu$ m diameter) is coupled to a microinterferometer (RICM). Since the time dependent particle-surface distance $h(t)$ and the equilibrium film thickness $h_{0}$ can be measured in thermal equilibrium, the interaction potential can be derived analytically from the Boltzmann probability distribution of the particle-surface distance $p(h): \Phi(h) / k T=-\ln [p(h)]+$ const.

\subsection{Viscoelastic properties of hydrated polymer films}

There are an increasing number of studies postulating that the accumulation of hyaluronic acids in an extracellular matrix plays an important role in the early stage of neuronal morphogenesis [1]. Many of their unique biological functions originate from the viscoelastic nature of polymer networks, where polymers can include large amounts of solvents even at a small volume fraction. This is particularly important for understanding the lubricating properties of the extracellular matrix. As a preliminary attempt to study the interfacial interaction potential in these films in bulk water, we measure the viscoelastic properties of hydrated polysaccharide films by coupling a colloidal probe technique to a microinterferometer (reflection interference contrast microscopy, RICM; figure 6) [42]. A colloidal probe (a latex bead with 16-20 $\mu \mathrm{m}$ diameter) is used to determine the equilibrium film thickness with nanometre order accuracy. Since the system is in thermal equilibrium with the surrounding environment, the analysis of the vertical Brownian motion of a bead yields the interfacial interaction potential from the Boltzmann probability distribution of the particle-substrate distance $p(d)$ :

$$
\frac{\Phi(d)}{k T}=-\ln [p(d)]+\text { const. }
$$

Here, the characteristic mesh size that limits the hydrodynamic flow within the hyaluronic acid film is a function of the ionic strength and species. To achieve the film stability under water which is necessary for this series of experiments, hyaluronic acid molecules are randomly grafted via silane cross-linkers. The effect of monovalent ions on the swelling behaviour is described quantitatively by different theoretical approaches involving polyelectrolyte brushes [43,44] and neutral adsorbed polymers [45], demonstrating that it is possible to model the randomly grafted hyaluronic acid layer as a simple polyelectrolyte brush. On the other hand, this also means that this technique is not sensitive to the effect of the internal structures 

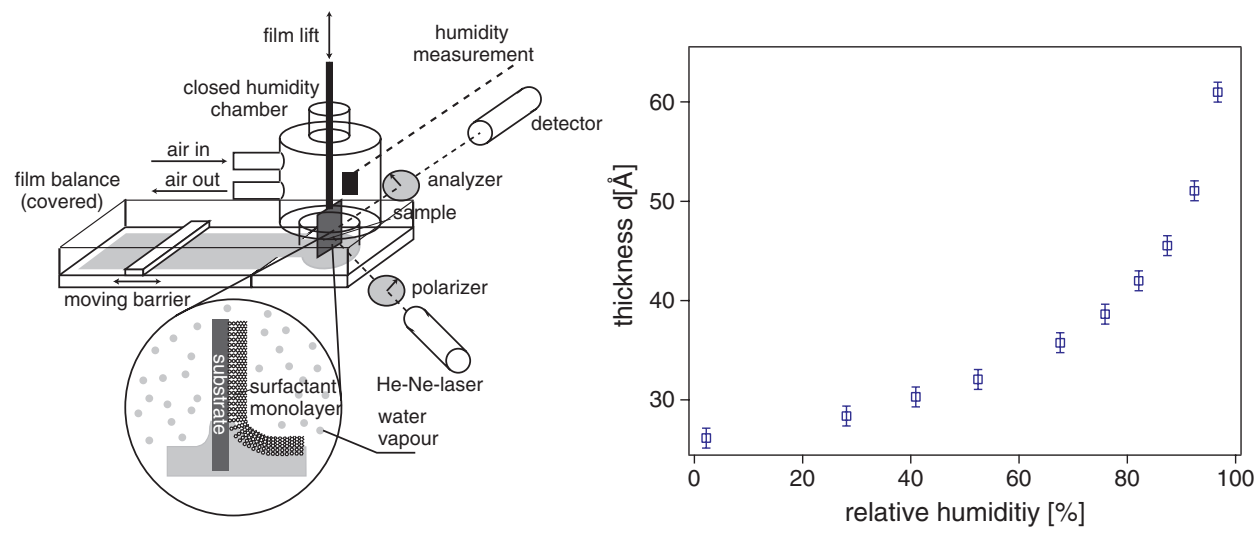

Figure 7. Left: a schematic drawing of an ellipsometer coupled with a Langmuir film balance, encapsulated in a climate chamber. The equilibrium thickness of the hydrated layer is measured at a defined disjoining pressure by subtracting the thickness of the alkyl layer from the total film thickness. Right: the hydration curve of a lipopolymer (with a PMOX33 head group) monolayer transferred at $30 \mathrm{mN} \mathrm{m}^{-1}$.

of the films on the general hydration. To gain more quantitative information about the local film structures, other techniques such as neutron reflectivity measurements will be required.

\section{Hydration and dehydration of glycocalix models}

\subsection{Hydration of glycolipid monolayers}

In this section, we describe our experimental approaches used to model the cell extracellular matrix interactions by looking at the system from the plasma membrane side (figure 1(a)). Glycocalices, oligosaccharide and polysaccharide chains of glycolipids, glycoproteins, and trans-membrane proteoglycans are localized in the outer leaflet of the lipid bilayer and stabilize the structure of plasma membranes by a combination of various physical forces (e.g. electrostatic force, long-range van der Waals interaction, hydrogen bonding) [2]. To understand the physical basis of structure-function relationships in glycocalix, we choose synthetic glycolipids and lipopolymers as the building blocks of the model glycocalix, and study the effects of molecular structures on their in-plane and out-of-plane cooperativity [46-50].

As the starting point, we study the lateral (in-plane) cooperativity in insoluble lipopolymer/glycolipid monolayers at the air/water interface under precise control of thermodynamic parameters using a Langmuir type film balance [46, 50-52]. Classical pressure-area isotherm measurements and fluorescence imaging allow us to quantify thermodynamic parameters (latent heat, phase transition entropy, critical pressure/temperature) as well as to observe phase separation and domain formations on the micron scale.

In the next step, monolayers of lipopolymers and glycolipids are transferred onto a solid surface by Langmuir-Blodgett (LB) deposition. Using the same strategy as described in section 1 (see figures 1 and 2), the swelling behaviour of carbohydrate head groups under defined osmotic pressure conditions is investigated by ellipsometry (figure 7) [39, 46, 51, 52]. LB deposition at a constant surface pressure enables us to control the surface density of hydrophilic chains (i.e., the area per molecule), and the ellipsometric measurements at well defined disjoining pressure allow the quantitative estimation of force-distance relationships within the hydrated head groups in the vertical (out-of-plane) direction. For the monolayer transferred at a high surface pressure $\left(\Pi>25 \mathrm{mN} \mathrm{m}^{-1}\right)$ corresponding to the liquid condensed 


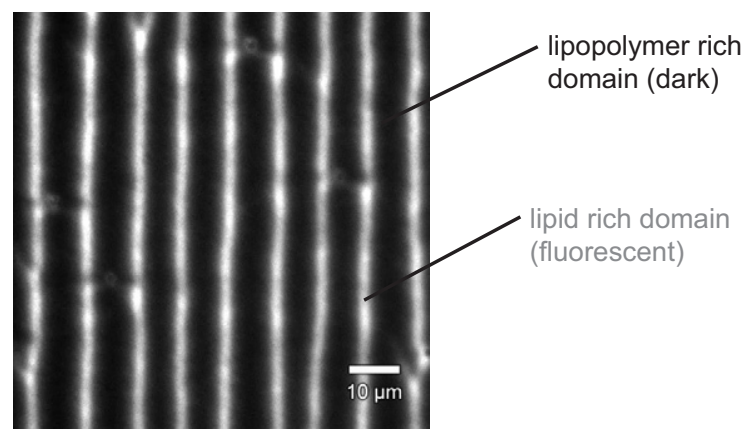

Figure 8. Dissipative stripe-like patterns of a lipid/lipopolymer monolayer (with a phytanoyl alkyl chain and PMOX12 head group) transferred at $\Pi=30 \mathrm{mN} \mathrm{m}^{-1}$ and a transfer velocity of $50 \mu \mathrm{m} \mathrm{s}^{-1}$. The lipid phase is labelled with $0.2 \mathrm{~mol} \%$ of Texas Red-PE lipid.

phase, the refractive index and thickness of the alkyl chains can be assumed to be almost constant and, therefore, the thickness of the hydrated layer can be determined.

\subsection{Dissipative structure formation induced by dewetting}

Lipids coupled to hydrophilic polymer head groups are expected to serve as 'spacer' molecules that increase the distance between supported lipid membranes and the surface. Recently, we established such 'polymer-tethered' lipid membranes with well defined poly(oxazoline) spacers $[52,53]$ following a two-step preparation:

(i) LB transfer of lipid/lipopolymer mixtures; and

(ii) deposition of the upper leaflet of the membrane by vesicle fusion.

While optimizing the preparation of polymer-tethered membranes, we observed stripe-like micropatterns of fluorescent lipid labels. Since no phase separation could be observed in monolayers at the air/water interface and the transfer efficiency (the ratio between the substrate area and the decrease in the area of the monolayer) remained 1.0, the results obtained implied demixing of lipid/lipopolymer mixtures. Selective labelling of lipids and lipopolymers confirms clear demixing of the two components, which is independent of the alkyl chains. The width and spacing between the stripes systematically decrease on increasing the transfer speed $[52,54]$. The observed demixing seems to be dependent on the velocity of water exclusion (dewetting) near the three-phase contact line (figure 8).

\section{Application of wetting principles in biological systems}

\subsection{Cell-cell contact as a wetting problem}

As mentioned in the previous sections, cell-cell contacts stabilized with hydrated biopolymer layers can be understood as an analogue of wetting (or spreading) of complex fluids on polymer films $[13,30,39]$. To keep a specific equilibrium distance between two neighbouring plasma membranes, the disjoining pressure in the biopolymer interlayer should fulfil the condition $\partial \Pi(d) / \partial d<0$. However, cell-cell contacts will be destabilized by the formation of tight local contacts, if the interaction is attractive, i.e. the disjoining pressure becomes negative. Furthermore, the wetting polymer layer should gain the surface free energy in the presence 

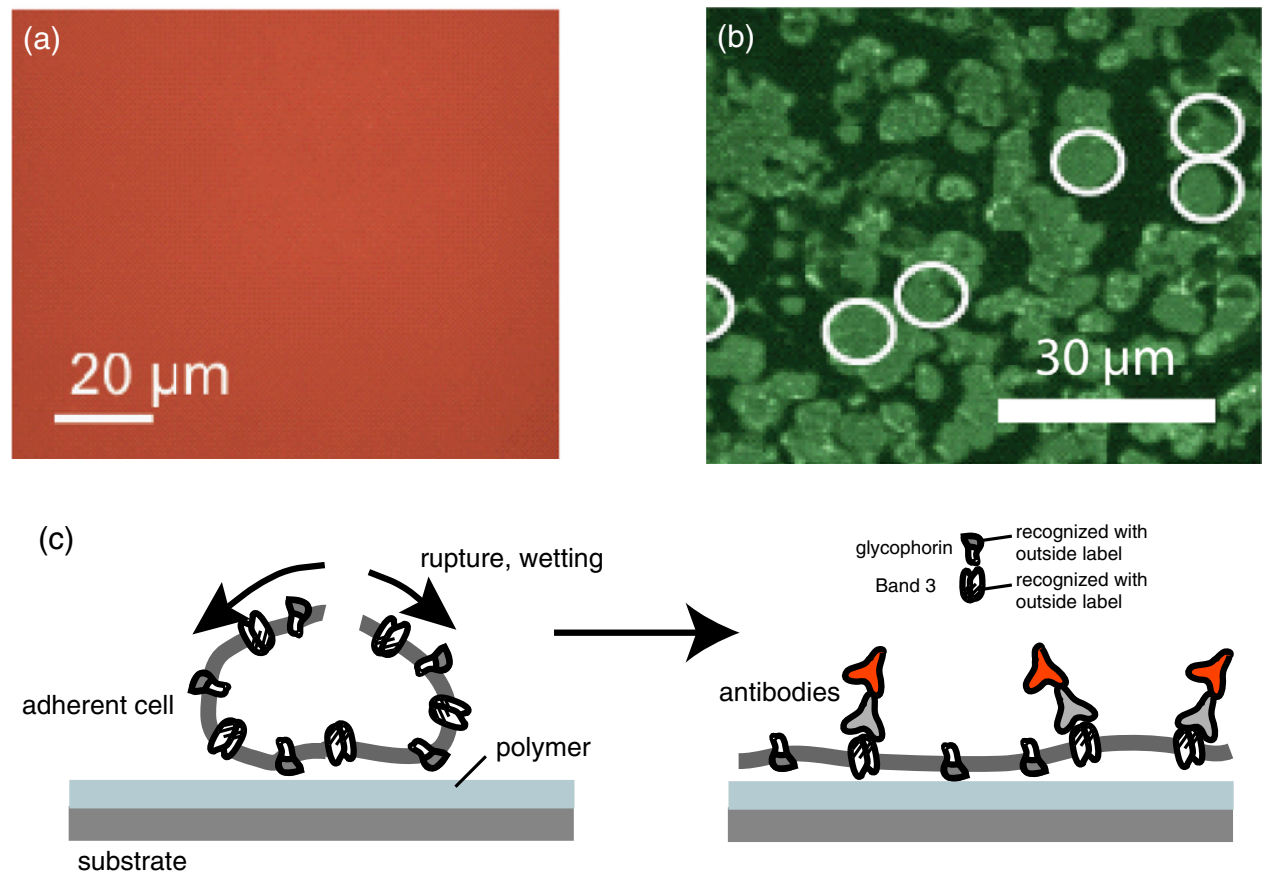

Figure 9. Immune-fluorescence (inside labelling) images of human erythrocyte membranes after incubation with a cellulose film (a) and a poly-lysine film (b). The outside labelling results in no fluorescence for both systems, confirming that all the adherent cells rupture and expose the cytoplasmic domain (inside) to bulk electrolyte (c).

of the neighbouring plasma membrane, which can be characterized with a positive spreading coefficient $S$ :

$$
S=\sigma_{\mathrm{SL}}-\sigma_{\mathrm{SM}}-\sigma_{\mathrm{ML}} \geqslant 0
$$

$\sigma_{\mathrm{SL}}, \sigma_{\mathrm{SM}}$, and $\sigma_{\mathrm{ML}}$ stand for the tensions at surface/liquid, surface/membrane, and membrane/liquid interfaces, respectively $[39,55]$.

\subsection{Selective wetting of cell membranes on biopolymers}

In our previous account, we reported the wetting of human red blood cell (erythrocyte) membranes on differently modified planar substrates [24]. Erythrocyte ghosts (red blood cells without the contents such as albumin) are prepared from freshly drawn blood as reported by Schwoch [56], and incubated for $60 \mathrm{~min}$ with the surface at room temperature $\left(\sim 20^{\circ} \mathrm{C}\right)$. After the incubation, the remaining ghosts and vesicles in the chamber were removed by intensive rinsing with buffer. Orientation of the erythrocyte membrane in contact with surfaces is identified with two immune-fluorescence labels: (1) the extracellular part of glycophorin can be labelled with a fluorescence labelled peanut agglutinin [24], or with a first monoclonal antibody (mouse $\mathrm{IgG}$ ) and a second polyclonal antibody (goat anti-mouse $\mathrm{IgG}$ ) labelled with dyes [57], while (2) the cytoplasmic domain of band 3 can be recognized with a first monoclonal antibody (mouse $\operatorname{IgG}$ ) and a fluorescence labelled second polyclonal goat anti-mouse IgG antibody (inside label). When the erythrocytes are incubated with planar glass cover-slides, no adsorption or rupture of erythrocytes is observed. This can be attributed to the repulsive 

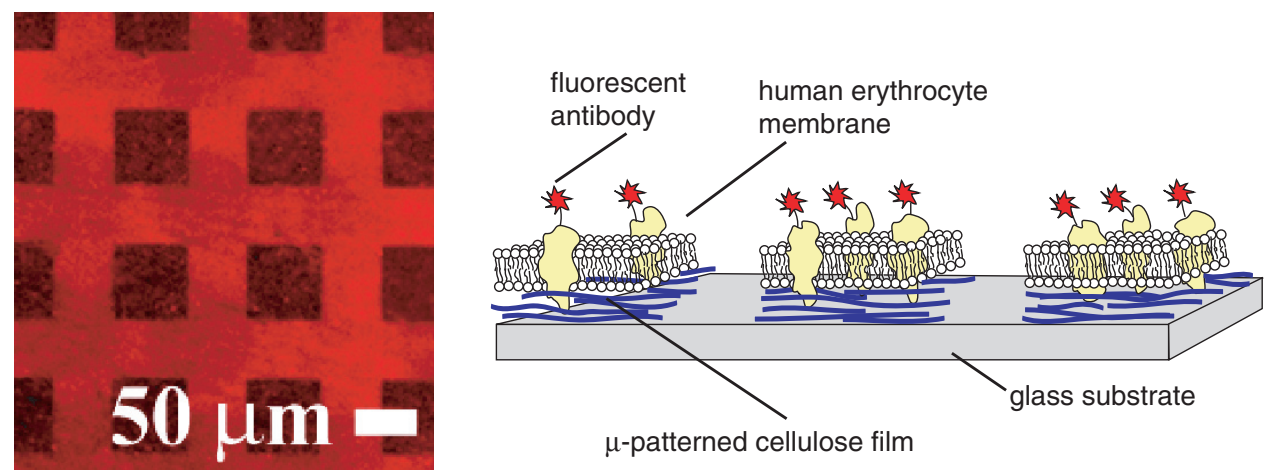

Figure 10. Selective wetting of human erythrocyte membranes on cellulose $\mu$ patterns $\left(d_{0}=5 \mathrm{~nm}\right)$ processed by deep UV photolithography.

interaction between the negatively charged cell glycocalix (owing to the sialic acid residues) and the negatively charged glass surface.

To optimize the interfacial interaction between cells and the surface, two kinds of hydrated polymer films are deposited on glass cover-slides: physisorbed films of cationic poly-lysine, and LB films of cellulose derivatives. In contrast to the results found for on glass surfaces, the inside labelling with antibodies indicates homogeneous wetting of erythrocyte membranes on cellulose surfaces, exhibiting no boundaries between individual patches of cell membranes (figure 9(a)). Since the outside labelling results in no fluorescence signal, we conclude that all the adherent cells rupture and expose their cytoplasmic domain to bulk electrolyte (figure 9(c)). The significant improvement in the wetting interaction for a thin polymer film with nm order thickness can be understood partially in terms of the reduction in electrostatic repulsion due to the deposition of a neutral polysaccharide layer that is thicker than the electrostatic screening length. Another possible contribution would be from the balance between the chemical potential of water in the hydrated cellulose layer and that in the cell surface glycocalix. To gain deeper insight into the wetting interaction, further quantitative measurements of hydration degrees (volume fractions of water) within each layer will be necessary. On the other hand, the inside labelling of the same cell membranes on poly-lysine shows a very inhomogeneous surface coverage, which cannot be improved even by a prolonged incubation time or thermal annealing (figure 9(b)). The outside labelling shows no fluorescence over the whole area, confirming that the dark region in the figure corresponds to defects. This can be understood as the dewetting of cell membranes, because of the strong attraction between weakly anionic cell glycocalix and strong polycation (poly-lysine) surfaces, which might even result in a negative disjoining pressure.

The transformation of three-dimensional cells into quasi-two-dimensional films on ultrathin polymer film established here also possesses a large potential in biotechnological applications. As demonstrated above, the intactness of glass and the high affinity of cellulose to native cell membranes can be utilized for fabrication of micropatterns of cell membranes on structured cellulose films [57]. For example, lithographically micropatterned films of regenerated cellulose $\left(d_{0}=5 \mathrm{~nm}\right)$ can serve as biocompatible templates for selective wetting with native cell membranes in confined geometries (figure 10). Since the height deviation $(5-20 \mathrm{~nm})$ is much smaller than the width of each pattern $(\geqslant 5 \mu \mathrm{m})$, the surface heterogeneity can be treated as almost columnar. Membrane micropatterns retain their structure for more than a week, confirming their thermodynamic and mechanical stability. The fine-tuning of local wetting conditions can be applied in the design of microarrays of cell membranes to study the function of membrane proteins within the individual compartments. 


\section{Acknowledgments}

We thank A Förtig, L Pagnoni, and R Jordan for the synthesis of poly(oxazoline) derivatives, C Gege and R R Schmidt for the synthesis of glycolipids, and G Wegner for helpful suggestions relating to cellulose chemistry. We also thank S Kaufmann, A P Wong, and M Tutus for their contributions in membrane micropatterning. This work was supported by the Deutsche Forschungsgemeinschaft through the Priority Project 'Wetting and Structure Formation at Interface' (DFG Sa246/27 and Ta259/2). MT is grateful to the DFG for the habilitation fellowship (Emmy Noether programme) and to the Centre of Excellence of Kyoto University for hospitality.

\section{References}

[1] Comper W D 1996 Extracellular Matrix (Amsterdam: Harwood Academic)

[2] Gabius H J and Gabius S 1997 Glycoscience (Weinheim: Chapman and Hall)

[3] Derjaguin B V and Churaev N V 1987 Surface Forces (New York: Consultants Bureau)

[4] Derjaguin B V, Kabanov B N, Voropaye T N and Titiyevs A S 1964 J. Colloid Sci. 19113

[5] Barclay L M, Harringt A and Ottewill R H 1972 Kolloid Z. Z. Polym. 250655

[6] Churaev N V and Derjaguin B V 1985 J. Colloid Interface Sci. 103 542-53

[7] Clunie J S, Goodman J F and Symons P C 1967 Nature 2161203

[8] Lipowsky R and Seifert U 1991 Langmuir 7 1867-73

[9] Swain P S and Andelman D 1999 Langmuir 15 8902-14

[10] Vrij A 1966 Discuss. Faraday Soc. 4223

[11] Langevin D 1998 Curr. Opin. Colloid Interface Sci. 3 600-7

[12] Reiter G, Sharma A, Casoli A, David M O, Khanna R and Auroy P 1999 Langmuir 15 2551-8

[13] Sackmann E and Bruinsma R F 2002 Chem. Phys. Chem. 3 262-9

[14] Albersdörfer A, Feder T and Sackmann E 1997 Biophys. J. 73 245-57

[15] Bell G I, Dembo M and Bongrad P 1984 Biophys. J. 451051

[16] Torney D C, Dembo M and Bell G I 1986 Biophys. J. 49 501-7

[17] Marx S, Schilling J, Sackmann E and Bruinsma R F 2002 Phys. Rev. Lett. 88138102

[18] Löfas S and Johnson B 1990 J. Chem. Soc. Chem. Commun. 21 1526-8

[19] Harris J M 1992 Poly(ethylene glycol) Chemistry (New York: Plenum)

[20] Israelachvili J N 1992 Intermolecular and Surface Forces 2nd edn (London: Academic)

[21] Mathe G, Albersdörfer A, Neumaier R and Sackmann E 1999 Langmuir 15 8726-35

[22] Rehfeldt F, Pagnoni L, Jordan R and Tanaka M 2002 Langmuir 18 4908-14

[23] Elender G, Kühner M and Sackmann E 1996 Biosens. Bioelectron. 11 565-77

[24] Tanaka M, Kaufmann S, Nissen J and Hochrein M 2001 Phys. Chem. Chem. Phys. 3 4091-5

[25] Rehfeldt F and Tanaka M 2003 Langmuir 19 1467-73

[26] Rand R P 1981 Ann. Rev. Biophys. Bioeng. 10 277-314

[27] Parsegian V A, Fuller N and Rand P 1979 Proc. Natl Acad. Sci. USA 76 2750-4

[28] Rau D C and Parsegian V A 1990 Science 249 1278-81

[29] de Gennes P G 1985 Rev. Mod. Phys. 57 827-63

[30] Leger L and Joanny J F 1992 Rep. Prog. Phys. 55 431-86

[31] Andelman D, Joanny J F and Robbins M O 1988 Europhys. Lett. 7 731-6

[32] Wolansky G and Marmur A 1998 Langmuir 14 5292-7

[33] Swain P S and Lipowsky R 1998 Langmuir 14 6772-80

[34] Groves J T, Ulman N and Boxer S G 1997 Science 275 651-3

[35] Nissen J, Jacobs K and Radler J O 2001 Phys. Rev. Lett. 86 1904-7

[36] Wiegand G, Jaworek T, Wegner G and Sackmann E 1997 J. Colloid Interface Sci. 196 299-312

[37] Wiegand G, Jaworek T, Wegner G and Sackmann E 1997 Langmuir 13 3563-9

[38] Mooney J F, Hunt A J, McIntosh J R, Liberko C A, Walba D M and Rogers C T 1996 Proc. Natl Acad. Sci. USA 93 12287-91

[39] Elender G and Sackmann E 1994 J. Physique II 4 455-79

[40] Albersdörfer A, Elender G, Mathe G, Neumaier K R, Paduschek P and Sackmann E 1998 Appl. Phys. Lett. 72 2930-2

[41] Neumaier K R, Elender G, Sackmann E and Merkel R 2000 Europhys. Lett. 49 14-9

[42] Albersdörfer A and Sackmann E 1999 Eur. Phys. J. B 10 663-72 
[43] Pincus P 1991 Macromolecules 24 2912-9

[44] Ross R S and Pincus P 1992 Macromolecules 25 2177-83

[45] de Gennes P G 1987 Adv. Colloid Interface Sci. 27 189-209

[46] Schneider M F, Mathe G, Tanaka M, Gege C and Schmidt R R 2001 J. Phys. Chem. B 105 5178-85

[47] Schneider M F, Lim K, Fuller G G and Tanaka M 2002 Phys. Chem. Chem. Phys. 4 1949-52

[48] Schneider M F, Zantl R, Gege C, Schmidt R R, Rappolt M and Tanaka M 2003 Biophys. J. 84 306-13

[49] Tanaka M, Schneider M F and Brezesinski G 2003 Chem. Phys. Chem. 4 1316-22

[50] Tanaka M, Schiefer S, Gege C, Schmidt R R and Fuller G G 2004 J. Phys. Chem. B 108 3211-14

[51] Mathe G, Gege C, Neumaier K R, Schmidt R R and Sackmann E 2000 Langmuir 16 3835-45

[52] Purrucker O, Förtig A, Jordan R and Tanaka M 2004 Chem. Phys. Chem. 5 327-35

[53] Förtig A J R, Purrucker O and Tanaka M 2003 Polym. Preprints 44850

[54] Purrucker O, Förtig A, Lüdke K, Jordan R and Tanaka M 2004 submitted

[55] Brochard-Wyart F and de Gennes P G 1992 Adv. Colloid Interface Sci. 39 1-11

[56] Schwoch G and Passow H 1973 Mol. Cell. Biochem. 2 197-217

[57] Tanaka M, Wong A P, Rehfeldt F, Tutus M and Kaufmann S 2004 J. Am. Chem. Soc. 126 3257-60 